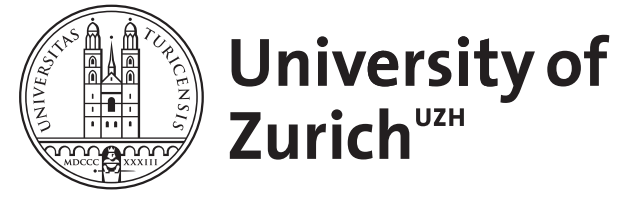
Archive

University of Zurich

University Library

Strickhofstrasse 39

CH-8057 Zurich

www.zora.uzh.ch

Year: 2017

Defining MoRAL After Liver Transplantation

Clavien, Pierre A ; Dutkowski, Philipp ; Lillemoe, Keith D

DOI: https://doi.org/10.1097/SLA.0000000000002008

Posted at the Zurich Open Repository and Archive, University of Zurich

ZORA URL: https://doi.org/10.5167/uzh-139110

Journal Article

Published Version

Originally published at:

Clavien, Pierre A; Dutkowski, Philipp; Lillemoe, Keith D (2017). Defining MoRAL After Liver Transplantation. Annals of Surgery, 265(3):555-556.

DOI: https://doi.org/10.1097/SLA.0000000000002008 


\title{
Defining MoRAL After Liver Transplantation
}

\author{
Pierre A. Clavien, MD, PhD, ${ }^{*}$ Philipp Dutkowski, MD, ${ }^{*}$ and Keith D. Lillemoe, MD $\dagger$
}

$\mathrm{R}^{\mathrm{c}}$ ecently, a new score was proposed in this Journal ${ }^{1}$ for identifying liver transplant HCC candidates with low risk of recurrence after transplantation, despite classified beyond the widely accepted Milan criteria. This score was nicknamed "MoRAL"1 to stand for model for tumor recurrence of after living donor liver transplantation (MoRAL). ${ }^{1}$ This scoring system combined serum $\alpha$ fetoprotein (AFP) levels with another marker "prothrombin induced by vitamin K absence II (PIVKA-II)" enabling to identify significant longer recurrence-free survival in a large series of living donor liver transplants with a c-statistic of 0.88 at a cut-off of 314.8. The MoRAL score formula $(=11 \times \sqrt{P I V K A}+2 x \sqrt{A F P})$ appears rather simple, but with a need for further validation, particularly in Western cohorts. ${ }^{2}$

The term MoRAL score has been introduced some years ago by the Columbia group ${ }^{3}$; at that time standing for Model of Recurrence After Liver transplantation. This original MoRAL score was, however, based on a different formula including AFP and neutrophil-to-lymphocyte ratio (NLR), instead of PIVKA-II. The formula was designed to stratify the risk of tumor recurrence after liver transplantation in patients with HCC.

In the current issue of the Journal, the same group now shows, mainly using cadaveric liver grafts, high accuracy of their original formula in predicting tumor recurrence after transplantation by combining AFP $>200$, NLR $\geq 5$, and tumor size $\geq 3 \mathrm{~cm} .{ }^{4}$ Adding other parameters available after surgery, such as vascular invasion and type of tumor histology achieved an impressive c-statistics of 0.91 . The challenge, however, remains to define clinical thresholds when to say „no “, i.e. denying transplantation because of too high risk for tumor recurrence.

Both studies show that those patients with high MoRAL scores (cutoff $315^{1}$ or $10^{4}$ ) do poorly despite classified within Milan criteria (Table 1). In contrast, cases with low MoRAL scores showed excellent outcome, even though being far outside of Milan criteria (Table 1). Both studies, yet, clearly suffer from too small caseload and too many patients with advanced tumor stages. To get wide acceptance, both MoRAL scoring systems should therefore be validated in different populations, and their predictive value convincingly compared.

Another aspect is the easiness of pre-transplant determination of scores. For example, while calculation of NLR is readily available at most centers, PIVKA analysis is less popular in Western countries. On the other hand, in a recent systematic review, pretransplant PIVKA levels [or also named des-gamma-carboxy prothrombin (DCP)], besides AFP, and allelic imbalance in microsatellites in DNA of tumor tissue were related to tumor recurrence. ${ }^{5}$ This finding points towards the importance of further investigating useful combinations of promising prognostic biomarkers to predict worse outcome in an era of extreme scarce liver grafts.

\section{TABLE 1.}

\begin{tabular}{llccc}
\hline & $\begin{array}{c}\text { Initial MoRAL } \\
\text { score }^{\mathbf{4}}\end{array}$ & $\begin{array}{c}\text { 5y recurrence } \\
\text { free survival }\end{array}$ & $\begin{array}{c}\text { LDLT MoRAL } \\
\text { score }^{\mathbf{1}}\end{array}$ & $\begin{array}{c}\text { 5y recurrence } \\
\text { free survival }^{\text {sure }}\end{array}$ \\
\hline Within Milan & MoRAL $\leq 10^{4}$ & $90 \%$ & MoRAL $\leq 314.8^{1}$ & $85 \%$ \\
& MoRAL $>10^{4}$ & $45 \%$ & MoRAL $>314.8^{1}$ & $50 \%$ \\
Outside Milan & MoRAL $\leq 10^{4}$ & $80 \%$ & MoRAL $\leq 314.8^{1}$ & $70 \%$ \\
& MoRAL $>10^{4}$ & $40 \%$ & MoRAL $>314.8^{1}$ & $18 \%$ \\
\hline
\end{tabular}

\footnotetext{
From the *Department of Surgery and Transplantation, University Hospital Zurich, Switzerland; and †Massachusetts General Hospital, Department of Surgery, Boston, USA.

The authors report no conflicts of interest.

Reprints: Pierre-Alain Clavien, MD, PhD, Department of Surgery and Transplantation, University Hospital Zurich, Raemistrasse 100, CH-8091 Zurich, Switzerland. E-mail: clavien@access.uzh.ch.

Copyright (C) 2016 Wolters Kluwer Health, Inc. All rights reserved.

ISSN: 0003-4932/16/26503-0555

DOI: $10.1097 /$ SLA.0000000000002008
} 


\section{REFERENCES}

1. Lee JH, Cho Y, Kim HY, et al. Serum tumor markers provide refined prognostication in selecting liver transplantation candidate for hepatocellular carcinoma patients beyond the Milan criteria. Ann Surg. 2016; 263:842-850.

2. Olthoff KM, Goldberg DS. The MoRAL path beyond Milan [Editorial]. Ann Surg. 2016;263:851-852.
3. Brubaker W, et al. ACT Meeting 2013. Am J Transplant. 2013;71.

4. Halazun KJ, Najjar M, Abdelmessih RM, et al. Recurrence after liver transplantation for hepatocellular carcinoma: a new MORAL to the story. Ann Surg. 2016;265:557-564.

5. Pommergaard HC, Burcharth J, Rosenberg J, et al. Serologic and molecular biomarkers for recurrence of hepatocellular carcinoma after liver transplantation: a systematic review and meta-analysis. Transplant Rev (Orlando). 2016;30:171-177. 